\title{
Keeping It Real: From Faces and Features to Social Values in Deep Learning Algorithms on Social Media Images
}

\author{
Anja Bechmann \\ Aarhus University \\ anjabechmann@cc.au.dk
}

\begin{abstract}
This paper wants to supplement computational tests of deep learning vision algorithms with a sociologically grounded performance test of three widely used vision algorithms on Facebook images (Clarifai, Google Vision and Inception-v3). The test shows poor results and the paper suggests the use of a two-level labeling model that combines features with theoretically inspired accounts of the social value of pictures for uploaders. The paper contributes a suggestion for labeling categories that connects the two levels, and in conclusion discusses both advantages and disadvantages in accelerating user profiling through a better understanding of the incentives to upload images in the data-driven algorithmic society.
\end{abstract}

\section{Introduction}

Tech companies and in particular social media services are increasingly using Artificial Intelligence (AI) and deep learning algorithms to label pictures and recognize users with a view to predicting user needs more effectively. Status updates, comments and especially images and videos are unstructured in the sense that algorithms do not know in advance what kind of content is to be expected and why it is posted by the user. The majority of images today are social media images [1], and large numbers are uploaded to social media platforms such as Facebook, Instagram and Snapchat every minute worldwide. The need to understand these digital traces of the users is growing, leading the largest internet companies in the world to focus on visual images as input data in deep learning algorithms in order to profile the user and predict the user's needs. Most standard big data software has a built-in algorithm for picture recognition. These algorithms are now said to perform convincingly in understanding what is in the picture, but how well do they actually perform on social media pictures when we analyze them from a sociological and not a computational perspective? How well do algorithms capture what is on the picture and not least the social value of the picture? What are the potential outcomes of such algorithmic recognition in the light of user profiling?

The aim of this paper is to discuss the ability of deep learning algorithms to analyze social media pictures (exemplified by Facebook images) from a critical sociological perspective by reviewing three different neural network algorithms to understand and predict picture content. This will feed into the general discussion in the field of internet research on the use of such algorithms to profile users.

\section{Existing sociological studies on algorithms}

Existing studies on algorithms within media sociological studies have an important critical approach to how algorithms shape our society and the way we as humans are interpreted in the digital layer of our lives, often on a more general level than suggested in this paper [e.g. 2]. Studies are interested in algorithms as a cultural phenomenon and an underlying feature of society that has unintentional power-related consequences for society in the form of exploiting user data either in a privacy perspective [e.g. 3], as a surveillance mechanism [e.g. 4] or as information and communication filters [e.g. 5,6]. These bodies of literature offer interesting insights into the societal consequences of algorithms that form the starting point of this paper; but they contain little or no description of the actual algorithms and their performance, apart from classifying the algorithms as "black boxes".

Another direction within critical algorithmic studies aims to understand the potential discrimination and power structures in the algorithm itself by "deciphering them" [7]. Sandvig et al. [8] propose the application of algorithm "audits" as a method to create transparency in otherwise closed 
environments. They suggest that transparency is created through the use of different access and test methods ranging from code readings and scripting access to programmatically constructed traffic (sock puppet) and human crowdsourced testing (e.g. Mechanical Turk).

In line with these studies, this paper tries to test some of these algorithmic "boxes" in order to build a bridge between the important general theory and the specific deep learning algorithms and their performance in social media user profiling. However, whereas deciphering studies often look at the "model layer" [9] in the algorithms themselves, this paper looks at how they interpret the individual user through the "data layer" [9]. Therefore, the focus lies on the user as a data double rather than on the algorithm. Neural networks are famous for their learning ability; but they are closely connected to learning data, which is why the paper wants to look at data performance as a first step towards discussing the use of neural networks in decision-making.

New studies in social media retrieve data and use machine learning to detect patterns in data streams [e.g.10,11]. These studies often have methodological accounts of retrieval protocols and machine learning algorithms, but the primary focus is not on the performance of the algorithms, but on the findings they create. This paper draws on the tradition of "decipher" studies and seeks to help fill the gap between low-level empirically grounded methodological accounts and the generic critical sociologically based discussion within the field of internet research.

\section{Images and meaning}

To achieve these aims, the paper builds on a theoretical foundation of the human-technology nexus [12,13] as an integrated arena for modulation [14], and social images inscribe this integration through a visual representation of the human gaze and the material body. This means that I work from a phenomenological assumption that the picture is a trace of the individual in the world as a construction of being, and can therefore be interpreted as such [15]. In this sense, users encode meaning into the picture and algorithms ideally decode this meaning. The scientific field of machine learning strives to obtain a near human processing [16]. Deep learning tries to find and understand patterns in order to know them, and can interpret them when they reappear [17]. The paper will look at the performance of specific picture processing methods to evaluate this approach with a focus on the underrepresented, the outliers or "the monsters" [18] that give meaning to the normal patterns. Specifically, this will come into play in the performance measurement. What is systematically or unsystematically left out by the algorithms, what are they not capable of recognizing, and how could this knowledge be re-introduced into the algorithmic design when we focus on social value in combination with image features?

The most difficult element in designing deep learning algorithms involves providing a deep understanding of the picture. Identifying faces and features in the pictures is the standard within the discipline. This is very difficult in itself, but not enough to actually pinpoint what the intention by the uploader was and hence what the social value of the picture is. The assumption of this paper is that the social value of the picture is important when it is used for user profiling and predictions. How do we move from faces and feature recognition towards recognizing the social value of the image?

Rose [19] distinguishes between different modalities in image analysis: the production/caption, the audience (in this case algorithms), and the image itself. According to Rose, meaning in images is not often isolated to the identification of faces and features, but happens on a much more implicit and tacit level which is often revealed through qualitative analysis such as discourse analysis or through anthropological studies. Building on Rose, I therefore approach the level of meaning by dividing algorithmic image performance into two levels:

\section{Identifying features (objects, places, faces etc.)}

2. Identifying the social value of the picture for the uploader

At the first level, questions of inclusion and exclusion become relevant in identifying the features: What is foregrounded, what is in the center of the picture, the golden cut, what is acted upon, what is in focus, what are the lines in the picture like, and what about the colors? Some exclusions or failures to identify the features correctly are due to limited data input in the algorithms. Other algorithms are trained on the wrong dataset. It is the assumption of the paper that if we want to understand social media images, it is not beneficial solely to train the algorithm on images from websites or Imagenet (www.imagenet.com), because this would result in an excessively generalized semantic meaning by collapsing entities into categories that are not suited for social media $[20,21]$. The algorithms need to be adjusted according to the specific communicative context of social media, in which image norms and rules 
potentially have a specific meaning and contain a majority of images with specific feature patterns.

In order to approach the second level of the image analysis, we need to use specific knowledge of social media images and incentives for posting on social media in order to qualify an identification of the social value the picture represents for the uploader. The following section contains a short review of existing studies on social value derived from posting social media images in order to qualify this discussion.

\section{Social media images and social value}

Existing studies point to social capital, selfportraying/self-image, and memory as the three main reasons for posting social media content [22,23,24,25,26,27,1]. Scheufele \& Shah [28] separate social capital into three domains: interpersonal (social network interaction), intrapersonal (inner emotions \& self-satisfaction), and behavioral (participation in civic and political activities).

The maintenance of Facebook profiles by uploading content has been associated with a high level of self-satisfaction or the intrapersonal social capital category, and maintaining their profiles makes users feel more connected with their peers [23]. Stern $\&$ Taylor [28] show that $74 \%$ of the students they have studied reported that their Facebook profiles provided an accurate picture of themselves. However, corresponding with the earlier section on image analysis, self-portraying is often constructed indirectly according to Zhao et al. [30]. Their results show that self-identity is achieved through glimpses of personality and implicit communication using "clues" instead of direct descriptions. This is confirmed in later studies $[31,1]$ that show how "context collapse" is dealt with by posting posts/images with references and meanings that only a few selected in-group members understand. Selfportraying or "egocasting" [26] is not only achieved through such different implicit clues, but also through comments, likes and shares from the group. Egocasting leaves a greater impression if there are also endorsements and descriptions by online followers/friends, supplementing and enhancing the social desirability of the user [32,24].

In a study of students using Facebook by Zhao et al. [30], the average number of pictures uploaded to Facebook profiles was 88.4 (median=63.5). In the study they analyze identity claims on a continuum ranging between visual (implicit) over enumerative to narrative (explicit) ( p. 1824). The visual is described as the "self as social actor", and refers to "showing without telling" and "watch me and know me by my friends", and highlights the depth and extent of an individual's social ties. Miller et al. [1] also point out that users acknowledge their relationships to others by sending them images or tagging (on Facebook) friends in the picture. Ito [in 1] describes picture sharing as an "intimate visual co-presence" that creates a social awareness among users when they exchange perspectives on their everyday lives through images. The enumerative is described as "self as consumer" and connects to the "interests" category in the Facebook profile that show the "cultural self", the consumption preferences and (good) taste [30]. Last but not least, the narrative connects to the "first person self" in the "about me" section on Facebook, the self-narration in which users describe themselves directly to their networks. Zhao et al. [30] concludes that it is especially pertinent to show a socially desirable self that is "popular", "well-rounded" and "thoughtful" (bear in mind this is a study of students). Selfies often feature not only the user uploading, but also the user in a group of friends indicating social popularity [1].

A large cross-regional ethnographic study of social media usage [1] suggests that the incentives to post also encompass preserving and sharing memories and experiences (p. 156), as well as sharing the "everyday monotony of life" (p. 164). Based on inspiration from Bourdieu, Miller et al. refer to "sociograms", visual records of social roles and relations that are worth preserving such as weddings, childbirth, birthdays, graduation, holidays, trips, and new homes/jobs. However, such sociograms (p. 165) may also be an indicator of good taste or social status. According to Miller et al. [1], images can also express opinions, for instance through memes or metaphorical pictures. And they can also be shared to generate humor, jokes and laughter, which in turn present the person as funny. Metaphorical images include images that are designed to bully someone or comment on people in some way, according to Miller et al. [1]. In other words, the value of such picture is to confirm and strengthen in-group relationship by excluding certain other people.

The next section will account for cases and methods, and is followed by a section, which tries to turn knowledge of the theory on social value into a coding scheme that can be tested on the algorithms in the findings section and can potentially be introduced into more context-sensitive algorithmic designs. 


\section{Case study \& method}

The paper will approach the performance of deep learning image algorithms (also termed deep neural networks/DNN within computer vision) qualitatively by testing how a selection of algorithms performs on a small dataset of 166 pictures, my own Facebook albums. Why have I not chosen to test the algorithms on the large number of private images from thousands of users available in our research lab? The reason is two-fold. First of all, it is pertinent to consider ethical aspects and security when handling private pictures. Within the field of large-scale social media research, several unfortunate incidences of data leakage have led to the de-anonymization of data [e.g. 33]. As the data in many cases is uploaded to company servers in cloud solutions, I needed to own the data myself. The auto-ethnographically inspired approach [34] enabled me to consent to the procedure. In a way I was also inspired by the audit method [9], not in the form of a crowdsourced audit (which could be ethically problematic for people in the Mechanical Turk's pictures), but as a data double single-person test. Choosing this method makes me particularly vulnerable to the accusation that I am over-fitting the model to the data as the data is biased by regional and socio-demographic parameters. I therefore see the work in this paper as only a first step in the construction of a social media image algorithm, but it is a proper first-phase test to discuss the principles of deep learning algorithms and the construction of users based on algorithmic processing.

To test deep learning algorithms on image recognition, I wanted a sample of two algorithms: one from a specialized startup company, and one from an established large internet company. I also wanted the algorithms to be easily available (API) and free of charge for a small sample. The cases that met these criteria were the startup Clarifai (www.clarifai.com) and the established massive data company Google and their algorithm Google Vision in the cloud platform (https://cloud.google.com/vision/). Two obvious cases were not chosen: IBM Watson and Microsoft Vision. This was because their sampling criteria were similar to those of Google. Clarifai is documented on the website, and functions as a general model that tries to identify what is in the pictures using a variety of feature recognition tools. Additionally, developers can choose to use other specialized models trained on specific themed datasets such as NSFW (not safe for work), weddings, travel and food. For the experiment in this paper we have chosen to test against the general model, as we do not know beforehand what the pictures contain. Google Vision is a welldocumented algorithm [35] that combines six kinds of labels: entire image detection, text detection, face detection, geographical landmark detection, logo detection and safe search detection (e.g. violence or nudity). Both algorithms provide a relevance score from 0 to 1 depending on how confident the algorithm is in a certain label's ability to describe the picture ( 1 being fully confident). As both algorithms are cloud-based solutions that prevent researchers from analyzing personal images from research participants in retrieved datasets, I wanted to test an open-source algorithm on university servers as well. There are not many documented and pre-trained open-source, convolutional neural networks (CNN) on the market (OpenCV is not pre-trained); but Google has made the pre-trained inception-v3 algorithm available as part of their Tensorflow machine learning software package. We do not know what kind of data the closed and commercial algorithms are trained on, so we cannot know the total number of possible labels the algorithms can recognize in the images. However, the inception-v3 is trained on Imagenet pictures, and this collection has about 1,000 labels (image-net.org). For this paper we will test the performance of the inception-v 3 in the current state without training it on our own social media lab datasets in order to see if it provides the same or better result compared to the commercial and closed algorithms of Clarifai and Google Vision.

The data analysis will be executed in three steps. First, we will run the dataset through the three pretrained algorithms to retrieve the predicted labels and the confidence scores. Second, we will manually label the pictures, and third we will try to recognize the social value of the pictures manually and label them according to the field knowledge laid out in the prior section.

\section{Developing first iteration of a coding scheme to capture social value}

The difficult task of introducing world knowledge and social context into the algorithm begins with the definition of what exactly is social value and how algorithms progress from knowledge of features to the higher level of social value categories. In this section I will provide a first iteration on social value categories by revisiting the theoretical section. To implement these categories into the final layer of a deep neural network requires a training dataset which is manually labeled with parent-child relationships 
between features and values, and which is outside the scope of this paper although it will be the steering focus for future work. If we return to the theoretical section on social values, a first iteration can be inspired by a combination of existing categories and definitions, especially by Zhao et al. [30] and Scheufele \& Shah [28]. I propose the following coding scheme/labels as a first iteration:

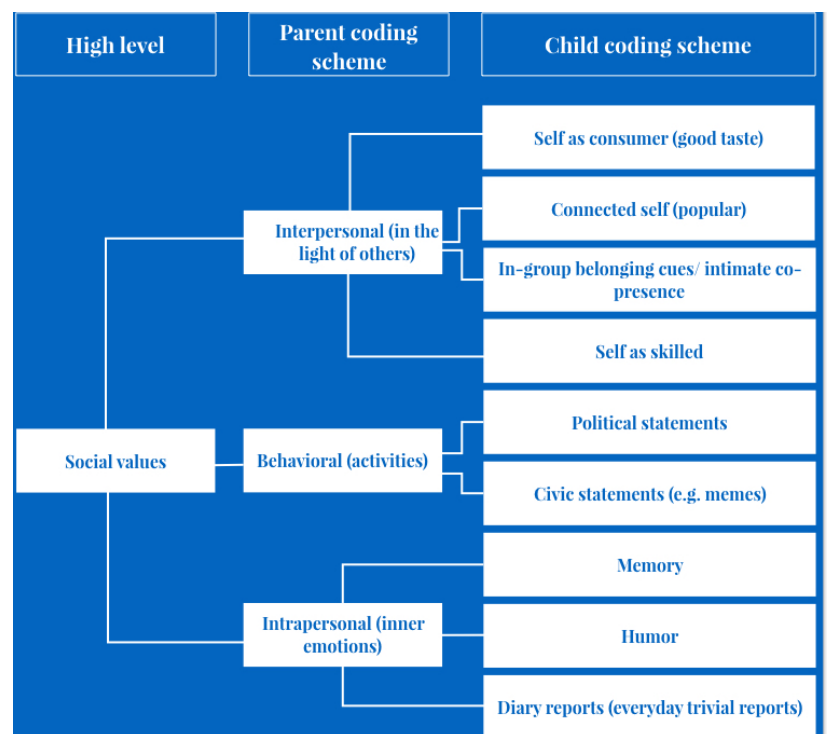

This is a parent-child coding scheme inspired by Zhao et al. and Scheufele \& Shah in particular. However, in combining the two studies I move away from Zhao's idea of a continuum between the toplevel categories of visual, enumerative and narrative. Their framework was on the basis of all communication and not specifically images. This is both an advantage and a disadvantage, as I suggest that all parameters can be present in images, including the visual, enumerative and narrative parameters inspired by for instance Rose's [19] interpretation of levels in image analysis. Hence, I remove this level as the top level and replace it with Scheufele \& Shah's categories (interpersonal, behavioral and intrapersonal) and instead use the next level to identify the child level and expand it to include supplementary knowledge and examples from social media studies [e.g. 1]. As this is a first iteration label suggestion, the paper will discuss experiences with the coding scheme in the conclusion with a special emphasis on overlapping or missing categories in the theoretically grounded coding scheme when it meets the empirical material. However, we need to account for potential overfitting in adjusting it according to the small dataset. The coding results will provide us with a basis for further developments of algorithms and for the discussion on how the algorithms take into consideration the social value, and what this means for user profiling on a more general societal level.

\section{Findings: Algorithmic performance on social media images}

Clarifai and Vision differ in terms of the number of unique labels and the average number of labels per picture (we were able to set the thresholds of inception-v3):

> Google Vision labels 165 pictures with 481 different unique labels. Average 6.6 labels per picture.

> Clarifai labels 165 pictures with 651 different unique labels. Average 20.0 labels per picture (they automatically provide the 20 most likely labels for each picture).

> In Inception-v3 we printed the 20 most confident labels. Inception-v3 labeled 165 pictures with 667 different unique labels. Average approx. 20 labels per picture (we set this limit to make it comparable to Clarifai instead of using the full variation of labels with very low confidence scores).

However, to account for the results of a sociologically grounded performance test I will use the manual coding of features as the baseline for the labels made by the three algorithms. With an intercoder reliability of $96 \%$ between manual coder 1 and myself, we were immediately confident about the relevant aspects of each picture for the uploader and the social context. Pictures of friends were the only pictures we did not label in the same way. Coder 1 does not know anything about these friend relations. In order to improve this, we could use the friend network to identify when friends are in the picture. In the following the performance of the algorithms is measured against manual coding labels semantically (instead of using the exact same words). The match in percentage is as follows when we look at the most confident labels from each of the three algorithms on the same 165 pictures:

\begin{tabular}{|l|l|l|l|}
\hline $\begin{array}{l}\text { Manual } \\
\text { coding }\end{array}$ & Clarifai & $\begin{array}{l}\text { Google } \\
\text { Vision }\end{array}$ & Inception-v3 \\
\hline $\begin{array}{l}100 \% \\
\text { (intercoder } \\
\text { reliability } \\
96 \%)\end{array}$ & $36 \%$ & $27 \%$ & $25 \%$ \\
\hline
\end{tabular}


In many instances, Clarifai provided generic labels with high confident scores such as people/no person, indoor and technology. Even though these are technically true, they do not give the content any meaning. I have only registered such generic categories as true if they give a picture meaning according to the manual coding. However, the result might have improved if we had tested the specialized packages as well. Google Vision provided fewer generic labels, but still it provided inaccurate descriptions of the central features. Inception-v3 seemingly has the lowest performance percentage. Even though this algorithm provided the most detailed labels, it also misinterpreted a large number of the pictures. This may be the result of pre-training on the Imagenet instead of using images bearing a greater resemblance to social media images. Overall the algorithms performed very poorly on this small Facebook dataset.

The algorithms all performed well on wedding pictures, food pictures and band pictures, but what kinds of features did the algorithms struggle to recognize in particular, and what could be the reason for this?

When it comes to social media research, Inception-v3 is of particular interest because it can run on university servers and thereby not violate user data privacy and security in future datasets with pictures not uploaded by me. I will therefore take a closer look at where the performance is weak and whether these weaknesses are systematic or not. The most consistent errors in the algorithm relate to pictures of children. This example illustrates the picture and the labels with the largest confidence scores.
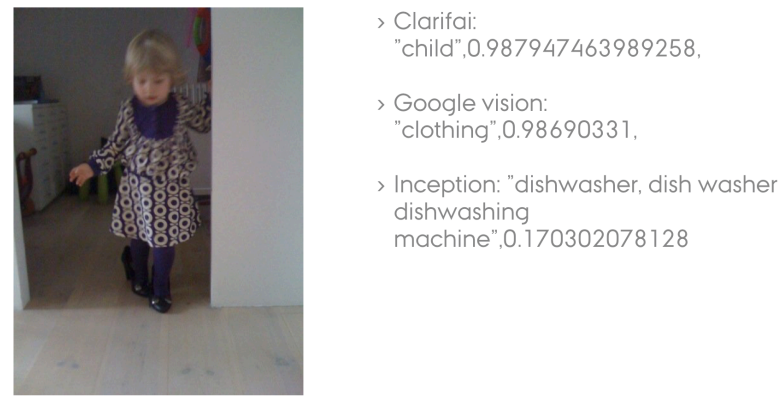

Inception-v3 seems to foreground the drawers in the background instead of focusing on the object in the center. The algorithm can be used to allow for this in future work. Furthermore, the training data may contain few pictures of children. In the social media it is pertinent to train with pictures of children as they belong to everyday reports and provide both memories and social capital among parents. Another systematic error in the picture recognition is the lack of prioritization of text recognition over picture features. The text often acts as a pointer to what is essential in the picture in the social framework. In this example the algorithm interprets the silk shirt as a lab coat, presumably because the training data contains health sector pictures.

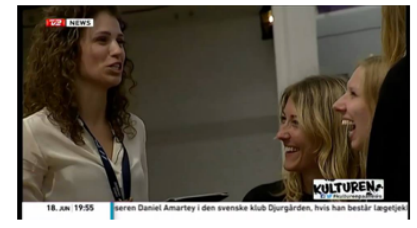

Clarifai:

"horizontal",0.990220308303833

Google vision: "person",0.8557657

Inception: "lab coat, laboratory coat", 0.413894712925

However, the interpretation lies in the text (TV station logo in the top left corner), which points to the national news setting. This in turn will improve the transition to the understanding of the social value of the picture. The same is the case with this book cover. The algorithm rightly suggests that it is a book jacket, but this is not the essence of the picture.
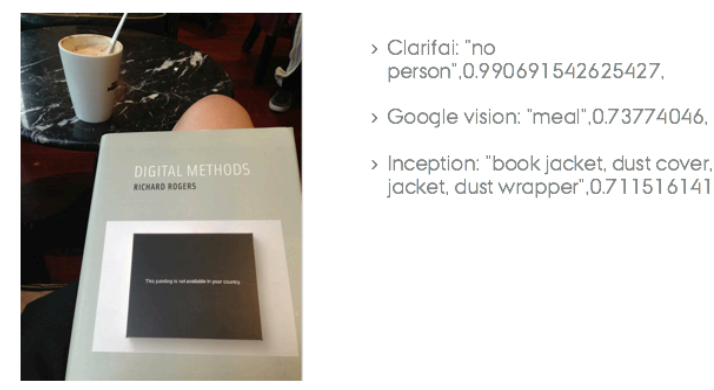

> Google vision: "meal",0.73774046,

Inception: "book jacket, dust cover, dust jacket, dust wrapper",0.711516141891

The essence is to show what kind of book I am reading. This would have been recognized by attaching more weight to the largest text in the center of the image.

When it comes to identifying social value, all the algorithms are blank. The obvious reason for this is that the algorithms are only trained on the feature level combined with text, face, landmark and logo detection. Nor are they trained specifically to recognize and understand social media images. However, we did not apply the additional trained algorithms to more specified features within the different packages. 
My argument in this paper is that by neglecting to train the algorithms on social values, the services that rely on the image prediction in relation to user profiling will have a less advanced suggestion for the user or make decisions on a less informed basis. In the case of food, this would mean that each time the algorithm detects a food-related picture it will suggest "self as consumer" as an "interpersonal" social capital label referring to the need to show "'good taste".

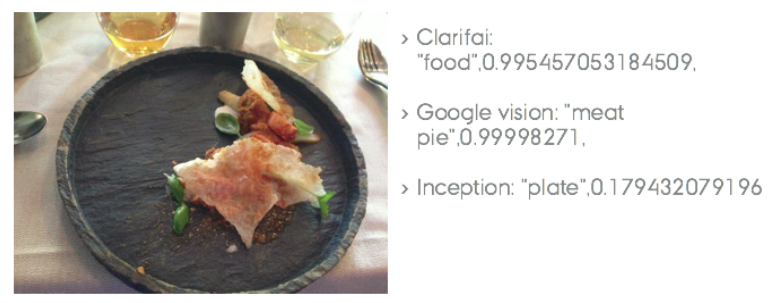

This would potentially allow for a more world- and context-sensitive approach to user profiling.

In the manual coding, coder 1 and I agreed about certain consistent patterns when identifying social value. For instance, food would connect to "self as consumer". Life events would connect to "memories". It would be interesting to focus on such consistent patterns in larger datasets in future research. All the labels on the social value level were used in the manual coding, but some pictures were difficult to label. This was especially the case with travel pictures. They were sometimes an indication of consumption (interpersonal), and sometimes an indication of memory (intrapersonal). Future work needs to detail this relation more and on a different and larger dataset.

\section{Conclusion: Deep learning algorithms and user profiling}

From the analysis of the three deep learning vision algorithms in this paper we have learned that for legal and ethical reasons we are not able to use cloud solutions such as Clarifai and Google Vision because these services require the users to upload pictures to company servers in order to improve their algorithm and potentially sell data. This is the standard procedure for open access vision algorithms at the moment (e.g. also IBM Watson).

Instead, we tried to test an open-source algorithm on university servers to see if it performed equally well. The paper found that from a qualitative, sociological point of view the algorithmic performance is very poor, but manual intercoder reliability was high. This indicates a potential for learning with new tagged social media training data as input in the neural network.

What, then, are the advantages and disadvantages of accelerating user profiling through a better understanding of the incentives to upload images in the data-driven algorithmic society?

In a society that increasingly relies on algorithmic decision-making, imprecise predictions in general can have an important negative impact on people's lives. For instance, if people are advised to watch totally irrelevant advertisements, presented with irrelevant content, if they are wrongly diagnosed, or even if they are denied access to bank loans and insurance policies based on predictions that use Facebook data (for instance). To improve predictions, I have suggested the inscription of two levels (parentchild) in the algorithmic labeling, a feature level and a social value level. By accounting for both the features and the social value of social media images, the accuracy of predictions might be improved. We need to work with this kind of inscription of context into the algorithm in collaboration between the computer and the social sciences. By recognizing existing theories as to why we post images, we open up for a broader understanding of the multiple level image analysis that is well-known within the field of communication studies and will hopefully be inscribed in a more nuanced way in future work on algorithms.

However, when we revisit the critical digital sociology literature the advantages of accuracy in predictions and the subsequent relevant decisionmaking are overshadowed by the disadvantages of such potential accuracy in terms of discrimination. This discrimination can take place by those holding massive data points, processing it and deciding on the algorithmic weights and thresholds [e.g. 8, 9, 6, 37].

Algorithmic discrimination happens both intentionally and unintentionally. Companies and regimes can intentionally control and punish unwanted behavior and preferences, but democratic societies can also adjust behavior through Foucauldian self-adjusted behavior in potentially surveilled environments or data-processing societies. Unintentional discrimination may occur (for instance) due to historical training data in societies where interpretations have developed over time, which is the case in the study of gender biases in word processing [37]. Unintentional discrimination is particularly relevant when we work with neural networks, multiple training datasets and multilevel processing, as it is very difficult for developers to create transparency if they are not held accountable 
through documentation/logs of every choice and every step they take.

This paper has tried to "decipher" vision algorithms through the data layer to critically access the performance and discuss the potential advances that could be made in user profiling. However, accuracy in data processing and user profiling on a general level becomes a question of societal values. What kind of profiling do we want to allow, and what kind do we not want?

\section{Acknowledgements}

Student developer Anders Geil for retrieving labels, student assistant Anne Hove Henriksen for helping with the manual coding of the pictures, Nicholas Wrigley for language assistance, and anonymous reviewers for great comments. Funding was gratefully received from the AUFF starting grant, DeiC and DighumLab.

\section{References}

[1] Miller, D., Costa, E., Haynes, N., McDonald, T., Nicolescu, R., Sinanan, J., Spyer, J. Venkatramen, S. \& Wang, X. (2016). How the world changed social media, London: UCL Press.

[2] Gillespie, T. (2014). The relevance of algorithms, in Gillespie, T., Bockowski, P. \& Foot, K. (Eds.). Media Technologies, Cambridge, MA: MIT Press.

[3] Cheney-Lippold, J. (2011). A new algorithmic identity: Soft biopolitics and the modulation of control, Theory, Culture and Society, 28 (6): 164-181.

[4] Introna, L. \& Wood, D. (2004). Picturing Algorithmic Surveillance: The Politics of Facial Recognition Systems, Surveillance \& Society, 2(2/3): 177-198.

[5] Bucher, T. (2012). Want to be on top? Algorithmic power and the threat of invisibility on Facebook. New Media \& Society, 14(7): 1164-1180.

[6] Bodle, R. (2015). Predictive Algorithms and Personalization Services on Social Network Sites: Implications for Users and Society, in Bechmann, A. \& Lomborg, S. (Eds.) The Ubiquitous Internet (eds. Bechmann, A. \& Lomborg, S.), New York: Routledge.

[7] Rieder, B. (2005). Networked Control: Search Engines and the Symmetry of Confidence, International Review of Information Ethics, 3(06): 26-32.

[8] Sandvig, C., Hamilton, K., Karahalios, K \& Langbort, C. (2014). Auditing Algorithms: Research Methods for Detecting Discrimination on Internet Platforms, Paper presented at $64^{\text {th }}$ Annual Meeting of the International Communication Association, May 22, Seattle, WA, USA.
[9] Diakopoulos, N. \& Koliska, M. (2016). Algorithmic transparency in the News Media, Digital Journalism, DOI: 10.1080/21670811.2016.1208053.

[10] Bruns, A. (2012). How long is a tweet? Mapping dynamic conversation networks on Twitter using Gawk and Gephi. Information, Communication \& Society, 15(9): 1323- 1351.

[11] Driscoll, K. \& Thorson, K. (2015). Searching and Clustering Methodologies: Connecting Political Communication Content Across Platforms. The ANNALS of the American Academy of Political and Social Science, 659(1): 134-148.

[12] Clark, Andy, (2011). Supersizing the mind: Embodiment, Action, and Cognitive Extension, New York: Oxford University Press.

[13] Haraway, D. (1991). A Cyborg Manifesto: Science, Technology, and Socialist-Feminism in the Late TwentiethCentury, in Simians, Cyborgs, and Women: the reinvention of nature. New York: Routledge.

[14] Star, S. L. (1991). Power, technology and the phenomenology of conventions: on being allergic to onions, in Law, J (ed) A Sociology of Monsters: Essays on Power, Technology and Domination, London: Routledge.

[15] Hall, S. (1993). Encoding/deconding in: The cultural studies reader (ed. During, S.), London: Routledge: 90103.

[16] Harnad, S. (2001). Minds, Machines, and Turing: The Indistinguishability of Indistinguishables, Journal of Logic, Language, and Information, 9(4): 425-445.

[17] Ananny, M. (2015). Towards an Ethics of Algorithms: Convening, Observation, Probability, and Timeliness, Science, Technology \& Human Values: 1-25. doi: $10.1177 / 0162243915606523$.

[18] Law, J. (1991). Introduction: Monsters, machines and sociotechnical relations, in Law, J (ed) A Sociology of Monsters: Essays on Power, Technology and Domination, London: Routledge.

[19] Rose, G. (2007). Visual methodologies: an introduction to interpretation of visual materials, London: Sage.

[20] Freedman, D. A. (2009). Statistical Models: Theory and Practice, New York: Cambridge University Press.

[21] Hastie, T., Tibshirani, R., \& Friedman, J. (2011). The Elements of Statistical Learning: Data Mining, Inference, and Prediction, Second Edition, New York, NY: Springer. 
[22] Lenhart, A. (2009) Teens and Social Media: An overview, Pew Internet Research report.

[23] Valenzuela, S., Park, N. \& Kee, K.F. (2009). Is There Social Capital in a Social Network Site? Facebook Use and College Students' Life Satisfaction, Trust, and Participation, Computer-mediated Communication, 14(4): 875-901

[24] Utz, S. (2010) Show me your friends and I will tell you what type of person you are: How one's profile, number of friends, and type of friends influence impression formation on social network sites, Computer-mediated Communication, 15(2): 314-335.

[25] Zhang, (2010). The Revolution will be Networked: The Influence of Social Networking Sites on Political Attitudes and Behavior, Social Science Computer Review, 28(1): 75-92.

[26] Hum, N.J, Chamberlin, P.E., Hambright, P.E., Portwood, A.C., Schat, A.C. \& Bevan, J.L. (2011) A picture is worth a thousand words: A content analysis of Facebook profile photographs, Computers in Human Behavior, 27(5): 1828-1833.

[27] Pempek et al., (2009). College students' social networking experiences on Facebook, Applied Developmental Psychology, 30: 227-238.

[28] Scheufele, D.A. \& Shah, D.V. (2000). Personality Strength and Social Capital: The Role of Dispositional and Informational Variables in the Production of Civic Participation, Communication Research, 27(2): 107-131.

[29] Stern \& Taylor (2007). Social networking on Facebook, JCSTAND: 9-20.

[30] S. Zhao, S. Grasmuch, J. Martin (2008). Identity construction on Facebook: Digital empowerment in anchored relationships, Computers in Human Behavior, 24, pp. $1816-1836$

[31] Marwick, A. \& boyd, d. (2011). Social Privacy in Networked Publics: Teens' Attitudes, Practices, and Strategies, Paper for discussion at the Privacy Law Scholars Conference on June 2, 2011 in Berkeley, CA.

[32] Tidwell, L.C. \& Walther, J. B. (2006). ComputerMediated Communication Effects on Disclosure, Impressions, and Interpersonal Evaluations: Getting to Know One Another a Bit at a Time, Human Communication Research, 28(3): 317-348.

[33] Zimmer, M. (2010). "But the data is already public": on the ethics of research in Facebook, Ethics information technology, 12: 313-325.

[34] Ellis, C., Adams, T. E. \& Bochner, A.P. (2011). Autoethnography: An overview, $F Q S, 12(1)$ :
[35] Casalboni, A. (2015). Google Vision API: Image Analysis as a Service, CloudAcademyBlog, http://cloudacademy.com/blog/google-vision-api-imageanalysis/.

[36] Bechmann, A. (2013). Internet profiling: The economy of data intraoperability on Facebook and Google, Mediekultur: Journal of Media and Communication Research, 55: 72-91.

[37] Bolukbasi, T., Chang, K.W., Zou, J, Saligrama, V. \& Kalai, A. (2016). Man is to computer programmer as woman is to homemaker? Debiasing word embeddings, Cornell University Library, http://arxiv.org/abs/1607.06520. 